Sādhanā, Vol. 19, Part 2, April 1994, pp. 289-309. (C) Printed in India.

\title{
Development of robust finite elements for general purpose structural analysis
}

\author{
G PRATHAP*, B P NAGANARAYANA and B R SOMASHEKAR \\ Structural Sciences Division, National Aerospace Laboratories, Bangalore, \\ 560017 , India \\ *Also, Jawaharlal Nehru Centre for Advanced Scientific Research, Indian \\ Institute of Science Campus, Bangalore 560012, India \\ MS received 20 August 1993; revised 18 February 1994
}

\begin{abstract}
The finite element method emerged out of the old work and energy methods and matrix structural analysis to become a numerical procedure to solve practical stress analysis problems in solid and structural mechanics. With the impetus given by the rapid development of computer technology, it became the most overwhelmingly popular analysis and design computational tool for a very wide spectrum of engineering science, e.g. fluid mechanics, heat transfer and electro-magnetics. Today, there are very powerful general-purpose software codes that make analyses and design tasks that were once considered to be intractable, routinely simple. Many of these are closely held proprietary codes owned and used in-house by large engineering firms or sold or licensed and supported by specialist companies. (Recent estimates indicate that the market for these codes has reached a turnover of a billion dollars and that industries and institutions spend several tens of billions of dollars in running such codes.) These codes are rarely given out in source code. In order to have an in-house code that could be continuously up-graded and enhanced, NAL initiated some work to develop a medium-sized general purpose code (about 20,000 lines of FORTRAN code) for the analysis of laminated composite structures (FEPACS - finite element package for analysis of composite structures), recognising the importance that laminated composites were assuming in aerospace structural technology.
\end{abstract}

Several key elements commonly found in general purpose packages (GPP) used by the aerospace, automobile and mechanical engineering industries were identified. These were re-designed incorporating anisotropic composite capabilities and validated. Many hurdles were faced during this task and required an examination of the basic issues at a paradigmatic level. Concepts such as consistency and variational correctness were introduced and studied critically. These guidelines played a critical role in developing robust versions of the elements and are briefly covered in this review. The paradigms also helped to identify procedures to perform 
a priori error estimates for the quality of approximation and this allowed the elements being developed to be critically validated.

The article concludes with a summary of what has been achieved and also suggests areas where the concepts can be applied fruitfully in the study of the displacement type finite element method.

Keywords. Finite element method; composite structures; structural analysis; element technology; general purpose packages; FEPACS.

\section{Introduction - from $C^{1}$ to $C^{0}$ elements}

We shall examine the subject as seen from our own practical viewpoint and as it must have been seen by the larger finite element community as well, as we undertook the task of designing a library of simple, accurate and efficient elements for general purpose finite element structural analysis.

At the time we began our work, around 1978, it was slowly becoming accepted that the element libraries of major general purpose packages (GPP) were replacing what were called the $C^{1}$ elements with what were known as the $C^{0}$ elements. The former were based on well-known classical theories of beams, plates and shells (i.e. the Euler-Bernoulli beam theory, the Kirchhoff-Love plate theory and the equivalent shell theories), reflecting the confidence that structural analysts have had in such theories for over two centuries. It is indicative that the early history of the finite element technology was almost entirely confined to the use of elements based on such theories. These theories did not allow for transverse shear strain and permitted the modelling of such structures by defining deformation in terms of a single field, $w$, the transverse deflection of a point on what is called the neutral axis (in a beam) and neutral surface of a plate or shell. The strains could then be computed quite simply from the assumption that normals to the neutral surface remained normal after deformation. One single governing differential equation resulted, although of a higher order (in comparison to other theories we shall discuss shortly), and this was considered to be an advantage.

There were some consequences arising from such an assumption both for the mathematical modelling aspect as well as for the finite element (discretisation) aspect. In the former, it turned out that to capture the physics of deformation of thick or moderately thick structures, or the behaviour of plates and shells made of newly emerging materials such as high performance laminated composites, it was necessary to turn to more general theories accounting for transverse shear deformation as well - these required the definition of rotations of normals which were different from the slopes of the neutral surface. Some of the contradictions that arose as a result of the old $C^{1}$ theories - e.g. the use of the fiction of the Kirchhoff effective shear reactions could now be removed, restoring the more physically meaningful set of three boundary conditions on the edge of a plate or shell (the Poisson boundary conditions as they are called) to be used. The orders of the governing equations were correspondingly reduced. A salutary effect that carried over to finite element modelling was that the elements could be designed to have nodal degrees of freedom which were the six basic engineering degrees of freedom - the three translations and the three rotations at a point. This was ideal from the point of view of the organization of a general purpose package. Also, elements needed only simple basis functions requiring only 
the continuity of the fields across element boundaries - these are called the $C^{0}$ requirements. In the older $C^{1}$ formulations, continuity of slope was also required and to achieve this in arbitrarily oriented edges, as would be found in triangular or quadrilateral planforms of a plate bending element, it was necessary to retain curvature degrees of freedom $\left(w_{x x}, w_{x y}, w_{y y}\right)$ at the nodes and rely on quintic polynomials for the element shape or basis functions. So, as general purpose packages ideal for production run analyses and design increasingly found favour in industry, the $C^{0}$ beam, plate and shell elements slowly began to replace the older $C^{1}$ equivalents. It may be instructive to note that the general two-dimensional (i.e. plane stress, plane strain and axisymmetric) elements and three-dimensional (solid or brick as they are called) elements were in any case based on $C^{0}$ shape functions - thus this development was welcome in that universally valid $C^{0}$ shape functions and their derivatives could be used for a very wide range of structural applications.

However, surprisingly dramatic failures came to be noticed when $C^{0}$ elements were formulated. The greater part of academic activity in the late seventies, most of the eighties and even in the nineties was spent in understanding and eliminating what were called the locking problems. A good idea of the challenges involved can be seen in two recent reviews - a bibliography of the finite element formulation of constrained media elasticity (Prathap \& Nirmala 1990) - about 500 papers in thirty years, and a review of the quest for a reliable degenerate shell element (Gilewski \& Radwanska 1991 ) - over 350 papers in about three decades of activity.

These spectacular failures were called the 'locking' problems in $C^{0}$ finite elements. It was not clear why the displacement type method, as it was understood around 1977 , should produce for such problems, answers that were only a fraction of a percent of the correct answer with a practical level of discretisation. Studies in recent years have established that an aspect known as consistency must be taken into account.

The consistency paradigm requires that the interpolation functions chosen to initiate the discretisation process must also ensure that any special constraints that are anticipated must be allowed for in a consistent way. Failure to do so causes solutions to lock to erroneous answers. The paradigm showed how elements can be designed to be free of these errors. It also enabled error-analysis procedures that allowed errors to be traced to the inconsistencies in the representation to be developed. The authors have now developed a family of such error-free robust elements for application in structural mechanics and these are now available in a package, FEPACS (finite element package for analysis of composite structures), developed at the National Aerospace Laboratories.

This article would therefore review the understanding of such errors on a paradigmatic basis and the arrival at the end, of a family of robust elements of acceptable accuracy for use in typical GPP.

\section{Difficulties with $C^{0}$ elements: Locking and stress oscillations}

With the wide-spread acceptance of the $C^{0}$ family of elements, instances where the finite elements models of practical structures under certain physical conditions produced very erroneous solutions in spite of satisfying the continuity and completeness requirements came to be noticed (Doherty et al 1969; Pawsey \& Clough 1971; Zienkiewicz et al 1971). Such errors are today classified as locking - where errors in solutions grow indefinitely as the physical limits are approached (Prathap \& Bhashyam 1982) 
and delayed convergence - where the convergence rate of the solutions is much lower than that assured by the conventional continuity and completeness requirements of the finite element method (Prathap \& Babu 1986b). Such errors in displacement solution are always associated with violent stress oscillations (Prathap \& Babu 1987).

Walz et al (1970) classified errors into two categories - errors of first kind (errors due to the discretisation process but which disappear rapidly as mesh is improved) and errors of the second kind (discretisation errors which disappear very slowly and which get exaggerated when some structural parameter is changed). At the time this classification appeared (Walz et al 1970) it was not known that the latter class of errors were due to incorrect representation of the constrained strain energy components and arise purely from the way the finite element fields are expressed. Recent work shows that such errors become very serious in a particular class of problem - constrained media elasticity (Babu 1985; Prathap 1986, 1993; Naganarayana 1991). These problems span a wide range of structural phenomena - shear-flexible beams using Timoshenko theory (Prathap \& Bhashyam 1982) and plates/shells (Mindlin theory) suffering from shear locking (Hughes et al 1977; Prathap \& Viswanath 1983; Bathe \& Dvorkin 1985; Hinton \& Huang 1986; Donea \& Lamain 1987; Prathap \& Somashekar 1988), curved beam/shell structures suffering from membrane locking (Stolarski \& Belytschko 1981; Prathap 1985a, b; Prathap \& Babu 1986a; Jang \& Pinsky 1988), 2-D plane-stress, plane-strain and 3-D elasticity suffering from parasitic shear (Cook 1975; Prathap 1985c; Prathap et al 1986) and/or near incompressibility locking (Chandra \& Prathap 1989; Naganarayana \& Prathap 1991) etc. These are described in detail in table 1.

Many ad hoc techniques have been suggested to overcome such difficulties. Reduced/

Table 1. Some constrained-multi-strain problems in structural mechanics.

\begin{tabular}{|c|c|c|c|}
\hline \multirow{2}{*}{$\begin{array}{l}\text { Class of } \\
\text { structural } \\
\text { problem }\end{array}$} & \multicolumn{2}{|c|}{ Strain fields } & \multirow{2}{*}{$\begin{array}{l}\text { Type of constraints and } \\
\text { the associated penalty } \\
\text { limits }\end{array}$} \\
\hline & Unconstrained & Constrained & \\
\hline $\begin{array}{l}\text { Plane stress } \\
\text { Plane strain }\end{array}$ & Normal & Shear & $\gamma \rightarrow 0$ as $(b / l) \rightarrow 0$ \\
\hline $\begin{array}{l}\text { 3-D elasticity } \\
\text { in modes of } \\
\text { flexure }\end{array}$ & $\varepsilon$ & $\gamma$ & $\begin{array}{l}\text { where } b \text { and } l \text { form the } \\
\text { section on which } \gamma \text { is } \\
\text { acting s.t. } b<1\end{array}$ \\
\hline $\begin{array}{l}\text { Plane stress } \\
\text { Plane strain } \\
\text { 3-D elasticity } \\
\text { in modes of near } \\
\text { incompressibility }\end{array}$ & $\begin{array}{l}\text { Distortional } \\
\varepsilon_{d}\end{array}$ & $\begin{array}{c}\text { Dilatational } \\
\varepsilon_{v}\end{array}$ & $\begin{array}{l}\varepsilon_{v} \rightarrow 0 \text { as } \mu \rightarrow 0.5 \\
\text { for isotropic materials } \\
\text { where } \mu \text { is Poisson's } \\
\text { ratio }\end{array}$ \\
\hline $\begin{array}{l}\text { Shear-flexible } \\
\text { beam (Timoshenko) } \\
\text { plates (Mindlin) }\end{array}$ & $\begin{array}{c}\text { Bending } \\
\chi\end{array}$ & $\begin{array}{l}\text { Transverse } \\
\text { shear } \\
\qquad \gamma\end{array}$ & $\begin{array}{l}\gamma \rightarrow 0 \text { as }(t / l) \rightarrow 0 \\
\text { where } t=\text { thickness and } \\
l=\text { "element length" }\end{array}$ \\
\hline $\begin{array}{l}\text { Curved beams } \\
\text { and shells }\end{array}$ & $\begin{array}{c}\text { Bending } \\
\chi\end{array}$ & $\begin{array}{c}\text { Membrane } \\
\varepsilon\end{array}$ & $\begin{array}{l}\varepsilon \rightarrow 0 \text { as }\left(R t / l^{2}\right) \rightarrow 0 \\
\text { where } t=\text { thickness and } \\
R=\text { radius of curvature } \\
l=\text { "element length" }\end{array}$ \\
\hline
\end{tabular}


selective integration (Pawsey \& Clough 1971; Zienkiewicz et al 1971; Zienkiewicz \& Hinton 1976; Hughes et al 1977, 1978), assumed strain methods (MacNeal 1982; Oleson 1983), addition of bubble modes (Wilson et al 1973), residual energy balancing (Fried 1974, 1975), spurious mode decomposition (Belytschko et al 1984, 1985), discontinliaus force-field mixed methods (Noor \& Hartley 1977; Noor \& Anderson 1982; Noor \& Peters 1981), symbolic Fourier synthesis (Park 1984), unequal field interpolations with condensation of constraints (Tessler \& Dong 1981), Kirchhoff mode method (Stolarski et al 1985), quasi-conforming techniques (Tang et al 1984), use of trignometric basis functions (Hepper \& Hansen 1987), using shear constraints (Crisfield 1984) etc. represent a broad coverage of the various artifices used with varying degrees of success to resolve these issues. Often these procedures lacked an explanation for their success. Sometimes they were successful in one context and failed in some other. Again the reason for such behaviour was not clear.

Most explanations for the locking behaviour available at the time we started our work - singularity of shear stiffness (Zienkiewicz 1977), constraint counting and rank of the shear stiffness matrix (Cook et al 1981; Hughes 1987)-lacked a rigorous scientific basis. Zienkiewicz (1977) argued that the elements which lock have nonsingular shear stiffness matrices while the shear stiffness matrices of elements after reduced integration are singular. It was argued therefore that locking is due to non-singularity of the shear stiffness matrix and a reduced integration order that induces singularity in the shear stiffness matrix is recommended. In other words, the locking behaviour of an element is due to the high rank of the penalty-linked stiffness matrices. But, it was soon realised that an arbitrary reduction of the rank of the penalty-linked stiffness matrices may lead to the undesirable spurious zero energy mechanisms (Hughes 1987). The optimal rank for the shear stiffness matrix is often determined by a technique known as constraint counting (Malkus \& Hughes 1978). The method of constraint counting makes an attempt to determine optimal integration order based on number of constraints given in a problem. It is based on the ratio $r$ of the total active degrees of freedom in a given mesh $n$ to the total number of penalty constraints $m(r=n / m)$. Locking occurs if $r \leqslant 1$. The mesh does not lock if $r$ is slightly greater than unity and may have spurious zero energy modes if it is too high. Heuristically it argued that the near-optimal ratios are $r=2 / 1$ for 2-D problems and $r=3 / 1$ for 3-D problems (Cook et al 1981).

It should be observed here that such explanations are heuristic and lack a rigorous scientific basis i.e. the validity of the explanations is not numerically verifiable (falsifiable) since a causal relationship between the locking errors and the rank of the 'constrained' stiffness matrix is not established. Such arguments often follow from the given mesh for a structural problem rather than the basis of discretisation adopted in the finite element formulation. Such an explanation, hence, cannot be generally applied in developing a finite element. These arguments cannot identify the milder problems of delayed convergence which are observed in the case of higher order elements. Finally, they attempt explanations based on the symptoms accompanying the problem of locking (i.e. the high rank of non-singularity of the shear stiffness matrix is a symptom of an inconsistent formulation) rather than exploring the cause for the errors. There is no established procedure for obtaining the optimal rank of the penalty-linked stiffness matrices in literature. Here, we review some of the work done to provide a scientific basis for the origin of such errors. Very recently, it was possible to relate the consistency paradigm and the requirements that follow from it to the rank of the penality-linked stiffness matrix, showing that there is a link 
between the cause of such errors and the symptoms (i.e. high rank, non-singularity of matrices etc.) associated with the locking problem (Prathap 1994).

In the next sections, we shall present a scheme for error-free displacement type finite element formulation based on the consistency and correctness paradigms. The elements developed thus are now incorporated in an in-house finite element package called FEPACS.

\section{Field-consistency}

Prathap \& Bhashyam (1982) demonstrated that it is the inconsistent finite element representation of the constrained state of the strain energy in the respective penalty limits that causes problems like locking and stress oscillations. It considered a Timoshenko beam element formulation and showed that the constraint of vanishing transverse shear strain energy near vanishing beam thickness imposed two types of constraints. The constraints which had contributions from all the displacement fields appearing in the respective strain field definitions were classified as true constraints and the constraints that do not have contributions from at least one of the constituent displacement fields were classified as spurious constraints. It was also demonstrated that the latter (i.e. spurious constraints) disturbed the bending strain energy in the penality limits. Using this fact, analytical a priori error estimates were constructed to prove that it was the spurious constraints that caused locking and the associated stress oscillations (Prathap \& Babu 1986b, 1987). This was confirmed by conducting appropriate numerical experiments with the inconsistent finite element formulations which contain spurious constraints.

Eventually, the field-consistency paradigm emerged (Prathap 1986) to provide an explanation as well as a remedy for difficulties like locking and stress oscillations in the finite element formulation of the problems in constrained media elasticity. It holds the inconsistent representation of the constrained state of the corresponding strain energy components, giving rise to the spurious constraints in the penalty limits, responsible for such difficulties. It also suggests that the energy components associated with the spurious constraints have to be eliminated from the formulation for removal of the above-mentioned difficulties from the element formulation. The paradigm can be broadly stated as follows.

In a constrained media problem, some strains will have to vanish under certain conditions. Strain fields derived from displacement shape functions cannot always do this in a meaningful manner - spurious constraints are generated which cause locking. The consistency condition demands that the discretised strain field interpolations must be so constituted that it will enforce only physically true constraints when the discretised functionals for the strain energy of a finite element are constrained.

In the development of a finite element, the field variables are interpolated using interpolations of a certain order. From these definitions, one can compute the strain fields using the strain-displacement relations. These are obtained as interpolations associated with the constants that were introduced in the field variable interpolations. Depending on the order of the derivatives of each field variable appearing in the definition of that strain field (e.g. the shear strain in a Timoshenko theory will have $\theta$ and the first derivative of $w$ ), the coefficients of the strain field interpolations may have constants from all the contributing field variable interpolations or from only one or some of these. In some limiting cases of physical behaviour, these strain fields 
can be constrained to be zero values, e.g. the vanishing shear strain in a thin Timoshenko beam. Where the strain-field is such that all the terms in it (i.e. constant, linear, quadratic etc.) have, associated with it, coefficients from all the independent interpolations of the field variables that appear in the definition of that strain-field, the constraint that appears in the limit can be correctly enforced. We shall call such a representation field-consistent. The constraints thus enforced are true constraints. Where the strain-field has coefficients in which the contributions from some of the field variables are absent, the constraints may incorrectly constrain some of these terms. This field-inconsistent formulation is said to enforce additional spurious constraints.

We shall also determine procedures that can modify the element characteristics so that the consistency requirements are met. We shall call such elements the fieldconsistent elements as opposed to the field-inconsistent elements which do not take into account such requirements. There is a unique manner in which the field-consistent elements have to be generated - they have to satisfy a condition we shall call the correctness condition which will ensure that the variational theorems are not violated in the process of modifying the element stiffness matrix.

Scientific evidence and analytical proof for this paradigm have been provided and verified through several practical examples of finite element formulations over the years (Babu 1985; Prathap 1986, 1993; Naganarayana 1991). Using the so-called fieldreconstitution technique, analytical a priori estimates of the errors in displacement as well as stress recovery arising due to violation of various field-consistency requirements are derived for a family of elements and are digitally verified using appropriate computational models, see Prathap \& Nirmala (1990) for a bibliography.

Most of the ad hoc techniques mentioned before offer procedures for achieving field-consistency with varied degrees of success. The assumed strain methods appear to be the most versatile among these since they have the capability of isolating the spurious constraints in the formulation. Thus the behaviour of the inconsistent terms in the constrained strain fields and the associated spurious constraints becomes apparent and the construction of $a$ priori error estimates, with reference to displacement as well as stress recovery, becomes simplified. The assumed strain methods essentially try to eliminate the inconsistent terms in the original strain field (derived by the gradient operations on the kinematically admissible displacement fields) for the constrained strain component. The popular procedures used in the literature for achieving this are reduced/selective integration (Zienkiewicz \& Hinton 1976; Hughes et al 1978), least squares method (Bose \& Kirkhop 1984), mean value method (Donea \& Lamain 1987), collocation of the strain fields at certain standard points (Huang \& Hinton 1984; Bathe \& Dvorkin 1985) etc. and all these belong to assumed strain methods in a broad sense of definition.

Various aspects of the field-consistency paradigm are briefly illustrated using the simple example of a 2 -noded $C^{0}$-continuous Timoshenko beam element. The fieldreconstitution technique is used to derive a priori analytical error estimates for the additional stiffness parameter and stress oscillations which can be digitally verified. The technique is used to derive a priori error norms for many other useful elements in a series of publications.

\subsection{Example-linear Timoshenko beam element (BEAM2)}

A 2-noded beam element with two degrees of freedom (deflection $w$ and section rotation $\theta$ ) per node, shown in figure 1 , is considered. 


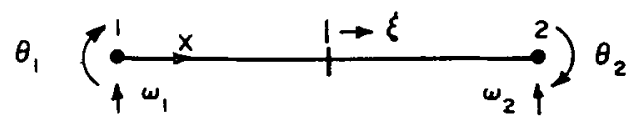

$$
\begin{aligned}
& L=\left(x_{2}-x_{1}\right) / 2 \\
& N_{1}=(1-\xi) / 2 \\
& N_{2}=(1+\xi) / 2
\end{aligned}
$$

Figure 1. 2-noded beam element.

Using the linear shape functions $N_{1}$ and $N_{2}$ the displacement fields can be expressed as,

$$
\theta=u_{0}+a_{1} \zeta \text { and } w=b_{0}+b_{1} \zeta
$$

Now, the two strain fields, flexure $\chi$ and the transverse shear $\gamma$, can be derived using (1) as

$$
\left\{\begin{array}{l}
\chi \\
\gamma
\end{array}\right\}=\left\{\begin{array}{c}
\mathrm{d} \theta / \mathrm{d} x \\
\theta-\mathrm{d} w / \mathrm{d} x
\end{array}\right\}=\left\{\begin{array}{c}
a_{1} / l \\
\left(a_{0}-b_{1} / l\right)+a_{1} \zeta
\end{array}\right\} .
$$

The strain energy can be considered as the sum of bending and shear strain energy components as follows.

$$
\begin{aligned}
U & =U_{B}+U_{S}=\int(E l / 2)(\chi)^{2} \mathrm{~d} x+\int(k G A / 2)(\gamma)^{2} \mathrm{~d} x, \\
& =(E I l)\left(a_{1} / l^{2}+(k G A l)\left\{\left(a_{0}-b_{1} / l\right)^{2}+\left(a_{1}\right)^{2} / 3\right\} .\right.
\end{aligned}
$$

In the penalty limits of vanishing beam thickness, the shear strain energy $U_{S}$ vanishes resulting in two constraints,

$$
\begin{aligned}
& a_{0}=b_{1} l \equiv \theta=\mathrm{d} w / \mathrm{d} x, \\
& a_{1}=0 \equiv \mathrm{d} \theta / \mathrm{d} x=0 .
\end{aligned}
$$

It is apparent that (4) is the true constraint representing the Euler-Bernoulli condition in the thin limits. But (5) is the spurious constraint which directly disturbs the bending strain energy. It can be noted that the spurious constraint corresponds to a situation where the beam is locked against rotation, thus explaining the locking behaviour of the problem. The field-consistency paradigm now suggests that the linear term in the shear strain field be eliminated for removal of locking and violent stress oscillations. The method of Legendre polynomial expansion can be used here so that the inconsistent linear term in shear strain is to be simply dropped. The procedure and variational justification for it are discussed in detail, in the next section.

It is also possible to obtain a priori error estimates by considering the strain energy after discretisation by a procedure called the functional reconstitution technique. For this example, it is possible to show that the effect of retaining the spurious constraint leads to an artificial stiffening of the bending action by a factor $\left(k G A l^{2} / 3 E I\right)$; i.e. the discretised beam behaves as if the moment of inertia of the cross section has changed from $I$ to $I^{\prime}$ such that,

$$
I^{\prime} / I=1+k G A l^{2} / 3 E l .
$$

Thus an analytical error norm for the additional stiffness parameter $e_{t h}$ can be written 
as,

$$
e_{t h}=I^{\prime} / I-1=k G A l^{2} / 3 E I
$$

This can be compared with results obtained from a actual finite element computation as,

$$
e_{\text {fem }}=\omega(t h) / \omega(\text { fem })-1
$$

The agreement of the computed errors (8) with the predicted errors (7) is very good and has been documented in Prathap \& Bhashyam (1982) and Babu (1985).

Through a similar exercise, in an inconsistent element with severe shear locking (when $e \gg 1$ ), the shear force resultant can be expressed as,

$$
Q=\bar{Q}+\left(3 M_{0} / l\right) \zeta
$$

where $\bar{Q}$ represents the correct shear force contributed from the consistent part of the shear strain field and $M_{0}$ is the constant part of the exact bending moment distribution. Thus the shear force recovered from the inconsistent element will have violent linear oscillations which are proportional to the constant part of the bending moment $M_{0}$. Again, the analytical predictions of the stress oscillations are well-matched by the finite element results (Babu 1985).

Such behaviour, i.e. locking and stress oscillations, is typical of inconsistently formulated beam, plate/shell, plane stress/plane strain and brick elements, the work horse elements of all current general purpose packages. It is imperative therefore that the design of a family of such elements must be carefully done to ensure that during formulation, the inconsistencies are systematically identified and removed so that the elements are free of such errors. The field-consistency paradigm offers us a conceptual scheme which enables this to be done.

\section{Variational correctness}

We now turn our attention to the task of formulating a consistent element without violating any of the norms required from energy and variational principles. It has become clear to us from the previous section that the field-consistency paradigm suggests the form of the interpolation functions for the constrained strain fields. However, the reconstituted assumed strain field interpolations cannot be chosen arbitrarily (as done, for example, in Mohr 1982). A variational basis for the correct field-redistribution (that is to find the coefficients of the consistent field from those of the original field) can be obtained by determining the conditions for exact equivalence of the assumed strain displacement approach to the mixed approaches based on the Hellinger-Reissner or $\mathrm{Hu}$-Washizu variational theorems (Simo \& Hughes 1986; Prathap 1988). The coefficients of the field-consistent assumed strain fields should now be determined from an orthogonality condition that arises when the equivalence of the minimum total potential energy principle with respect to the mixed variational principles is sought. This leads to another fundamental requirement in certain finite element formulations involving redistributed (or assumed) fields (e.g. strain or stress) variational correctness - that the redistributed field should be orthogonal to the error introduced because of the field-redistribution (Prathap 1988; Prathap \& Naganarayana 
1988; Naganarayana \& Prathap 1989a):

$$
\int \delta \bar{\gamma}(\bar{\gamma}-\gamma) \mathrm{d} x=0,
$$

where $\gamma$ represents the constrained strain field kinematically derived from the constituent displacement fields while $\bar{\gamma}$ is the assumed field to be determined from the original field such that $\bar{\gamma}$ is field-consistent and the resulting element formulation is free of locking and stress oscillations.

If the orthogonality condition that represents this equivalence is violated while designing the strain field to satisfy the field-consistency criteria alone, the resulting elements have poorer efficiency and are also plagued by undesirable strain and stress oscillations (Prathap \& Naganarayana 1988; Naganarayana \& Prathap 1989a).

In Prathap \& Naganarayana (1988), the variationally correct (or orthogonal) and incorrect (or non-orthogonal) field-consistent assumed strain forms of the quadratic and cubic shear deformable beam elements are used to explore these aspects in detail. It is shown that the non-orthogonal formulations can lead to reasonably accurate displacement solutions but have spurious stress oscillations. Using the fieldreconstitution technique, a priori analytical estimates were derived for the magnitude and pattern of these stress oscillations and tested digitally using computational results in Prathap \& Naganarayana (1988). These stress oscillations are related to the presence of artificially created spurious load mechanisms which are self-equilibrating.

These spurious load mechanisms lead to additional spurious linear oscillations in the shear force and bending moment in a quadratic Timoshenko beam element and spurious quadratic oscillations in bending moment (but no change in the shear forces) in cubic Timoshenko beam element as demonstrated in Prathap \& Naganarayana (1988). The resulting extraneous oscillations in the stress fields may often lead to difficulty in identifying points for optimal stress recovery in the element domain e.g. line-consistent 8-noded plate element (Naganarayana \& Prathap 1989a).

In many problems, it is possible to determine the variationally correct strain field by expanding the inconsistent field in terms of Legendre polynomials; it is easy then to identify and eleminate the inconsistent terms. The logic is simple and direct; the coefficients associated with each Legendre polynomial represents a discretised constraint in the penality limits; identify the inconsistent Legendre term applying the field-consistency paradigm and simply drop it to get the correct and consistent field. Since the Legendre polynomials are orthogonal in the domain of integration, it follows that this procedure satisfies the orthogonality condition arising from the equivalence of the minimum total potential energy principles with reference to the mixed principles and hence the method is variationally correct. The same result can often be achieved quite simply by using reduced integration if $\gamma$ is one order higher than $\bar{\gamma}$. It will be variationally incorrect otherwise. Reduced integration is universally popular since it is very easy to implement it on a computer. We shall see later that reduced integration cannot satisfy the edge-consistency requirements and hence fails when used in a distorted mesh. Hence the methods like Legendre polynomial expansion and truncation become very important in development of general purpose finite elements.

\section{Consistent non-uniform mapping and edge-consistency}

So far, we have looked at the consistency requirements in a simplified form that can be directly applied only to straight line elements or elements of rectangular form. It 
is not practical to have such a restriction for the plate/shell elements in a general purpose application. The development of an efficient and robust quadrilateral plate bending element has therefore been a formidable challenge. The point here is to ensure that consistency is maintained in curved and arbitrary quadrilateral forms of an element. The current practice in the development of elements is to use what is called the isoparametric concept - this requires the use of a covariant or natural co-ordinate system for all interpolations - i.e. the same interpolations serving to map both geometry (from the natural system to the Cartesian system) and displacements. The strains are based on the Cartesian system but interpolated using natural co-ordinates. It is therefore necessary to allow for changes from one system to another without loss of consistency, especially as far as the mapping of the constrained strain fields are concerned. It turned out that one crucial factor was the way tangential strain components on each edge of an element had to be defined under situations of non-uniform mapping.

That small errors in data preparation leading to distortion of the element can cause large errors in the solutions was reported by Hoppe (Hoppe 1984, 1985). This is due to the non-uniform mapping from the covariant system to the Cartesian system. The effect in the case of a constrained media problem is much more severe. Since curved beam, and quadrilateral plate and shell elements will intrinsically have such mapping conditions, there can be very large errors on this account. It is therefore necessary to take care to design an element so that accuracy and efficiency when used in a distorted mesh do not go down rapidly. There have been several attempts in literature to develop elements that are free of locking in their general form (Hughes et al 1977; MacNeal 1982; Hinton \& Huang 1986; Donea \& Lamain 1987) - most of them using the sampling points on the element edges. However, they lack an explanation for their success.

It was observed in Prathap \& Naganarayana (1992) that it is not sufficient if fieldconsistency is achieved in a variationally correct sense in the covariant natural system alone in such cases. It was demonstrated that the difficulties are with the non-uniform mapping of the strain fields because of which the consistency and correctness conditions achieved in the natural covariant system are not preserved over the element domain and across its boundary after transformation into the Cartesian system. Several methods of achieving consistent mapping of the constrained strain fields from the natural system to the Cartesian system were discussed from two popular points of view - Cartesian base formulation and covariant base formulation.

The additional requirement for an element to be free of errors in a patch is the edge-consistency requirement (Prathap \& Somashekar 1988). It was shown that the tangential strain components which are continuous across the element boundary in the undistorted covariant natural system should remain continuous even after the necessary transformations, and that the tangential strain components should be built from their corresponding tangential displacement components only. Though the Cartesian base formulation appeared to be very accurate in Prathap \& Naganarayana (1992), identification of tangential strain components and hence achieving edgeconsistency becomes a formidable job. Thus a covariant base formulation becomes desirable for developing general purpose finite elements.

We should note here that, in case of covariant base formulations, consistent mapping preserves the field-consistency over the element domain in the Cartesian system. However, special methods have to be used if edge-consistency has to be satisfied. An effective (from both accuracy and computational points of view) procedure namely, nodal Jacobian transformation, is developed and employed to 
achieve both consistent mapping and edge-consistency while developing several general purpose finite elements (Prathap \& Somashekar 1988; Prathap et al 1988; Naganarayana \& Prathap 1989a, 1989b; Naganarayana et al 1992; Prathap \& Naganarayana 1992). Such elements which satisfy both field- and edge-consistency requirements are subjected to several severe patch tests and are found to be very accurate.

It is interesting to observe here that the methods of sampling the constrained tangential strain components at the error-free points on the element boundary (Hughes et al 1977; MacNeal 1982; Hinton \& Huang 1986; Donea \& Lamain 1987) in fact, try to achieve field- and edge-consistency requirements in the Cartesian system in a similar fashion. This explains their success when used in a distorted mesh.

\section{Problems with initial strain/stress and varying moduli}

In problems with initial stress/strain or with varying moduli, the discretised stressresultant and strain fields will be of different order - consequently there is a loss of consistency in the formulation that results in oscillations in the stress-resultant fields (Prathap \& Naganarayana 1990a, and to be published; Naganarayana 1991). For example, it has been known for some time that thermal stresses computed directly from stress-strain and strain-displacement matrices in a finite element analysis with simple finite elements can show large errors (Ojalvo 1974; Pittr \& Hartl 1980). The conventional wisdom to tackle such problems is to sample stresses/strains at the Gauss points corresponding to a reduced integration rule. However, cases exist, e.g. the tapered quadratic bar element (Prathap \& Naganarayana 1990a), where the oscillations are such that easily identifiable points of accurate stress do not exist.

In the computation of the stiffness matrix, energy terms of the form $U=\int \sigma^{T} \in \mathrm{d} \Omega$ exist and if the order of $\sigma$ ( $\sigma$ may be stress or stress-resultant depending on the problem) is higher than that of the strain $\varepsilon$, the higher order term may not "do work" on the strain terms and are not recognised in the stiffness matrix. Thus, stress-resultants computed from the displacements recovered from such a formulation show extraneous oscillations.

It is necessary to define a consistent stress or stress-resultant field to ensure that the stress recovery reflects correctly the order of strain interpolations used. The orthogonality conditions, required for reconstituting the assumed fields for both strain and stress functions (from their original fields) simultaneously, can be obtained from the $\mathrm{Hu}-\mathrm{Washizu}$ variational principle as,

$$
\int \delta \bar{Q}(\bar{\gamma}-\gamma) \mathrm{d} \Omega=0 ; \quad \int \delta \bar{\gamma}(D \bar{\gamma}-\bar{Q}) \mathrm{d} \Omega=0,
$$

where, $\gamma, \bar{\gamma}, D \bar{\gamma}$ and $\bar{Q}$ represent the original inconsistent strain field, consistent strain field, stress-resultant field derived from the consistent strain field through constitutive laws and the consistent stress resultant field respectively.

In a varying moduli problem, due to varying $D$, the strain and stress-resultant fields will be of different interpolation order. In a thermal stress problem, the initial strain field in the element $\varepsilon_{0}$ which will be of the same order as the temperature field in the element, and the total strain field, which is obtained by differentiating the displacement fields will be of different order, especially if the temperature fields vary 
significantly over the domain and are interpolated by the same isoparametric functions as the displacement fields. Consequently, the total stress will be of an order higher than the strain field. Therefore, in recovering stresses in such problems, care must be taken to maintain consistency. In our work, the definitions of stress-resultants, or initial strains/stresses are a priori made consistent with the strain fields by invoking the orthogonality conditions seen earlier in this section.

\section{A complete consistent and correct procedure for displacement type finite element formulation}

So far, we have reviewed the important consistency and correctness paradigms necessary for finite element formulation free of locking and stress oscillations. Here, we sum up all these concepts and the additional considerations that are necessary for developing a general-purpose finite-element library for analysis of composite structures. For continuity, we recapitulate our understanding of the sources for the locking and stress oscillations and the required consistency and correctness paradigms to eliminate the same (table 2).

Continuity: The displacement function must be continuous over the element domain and across the element boundary in a given arbitrary finite element mesh.

Table 2. Types of errors, their sources in finite element analysis and the associated paradigms.

\begin{tabular}{|c|c|c|}
\hline Source & Symptoms & Paradigm/concepts \\
\hline $\begin{array}{l}\text { Finite element } \\
\text { discretisation }\end{array}$ & $\begin{array}{l}\text { Errors of first kind } \\
\text { Discretisation errors } \\
\text { (errors of first kind) }\end{array}$ & $\begin{array}{l}\text { Continuity and } \\
\text { Completeness }\end{array}$ \\
\hline $\begin{array}{l}\text { Constrained media } \\
\text { elasticity }\end{array}$ & $\begin{array}{l}\text { Errors of second kind } \\
\text { Locking, } \\
\text { delayed convergence, } \\
\text { stress oscillations }\end{array}$ & Field-consistency \\
\hline $\begin{array}{l}\text { Element distortion } \\
\text { (nonuniform } \\
\text { mapping) }\end{array}$ & $\begin{array}{l}\text { Locking, } \\
\text { delayed convergence, } \\
\text { stress oscillations }\end{array}$ & $\begin{array}{l}\text { Edge-consistency } \\
\text { and consistent } \\
\text { mapping }\end{array}$ \\
\hline Varying moduli & Stress oscillations & Stress field-consistency \\
\hline $\begin{array}{l}\text { Initial strain \& stress } \\
\text { field representation }\end{array}$ & $\begin{array}{l}\text { Strain and stress } \\
\text { oscillations }\end{array}$ & Stress field-consistency \\
\hline $\begin{array}{l}\text { Redistribution of } \\
\text { strain/stress fields }\end{array}$ & $\begin{array}{l}\text { Poorer convergence } \\
\text { spurious load } \\
\text { mechanisms and } \\
\text { stress oscillations }\end{array}$ & $\begin{array}{l}\text { Variational } \\
\text { correctness }\end{array}$ \\
\hline $\begin{array}{l}\text { Modelling warped } \\
\text { Surface with } \\
\text { linear elements }\end{array}$ & $\begin{array}{l}\text { Erroneous displacements } \\
\text { and stress recovery }\end{array}$ & $\begin{array}{l}\text { Warping } \\
\text { correction }\end{array}$ \\
\hline
\end{tabular}


Completeness: The strain/stress fields should be able to model strain-free rigid body motion of the element and the constant strain state of the element deformation.

Field-consistency: The terms in a constrained strain field that have partial contribution from the constituent displacement fields, leading to the spurious constraints in the penalty limits for the corresponding strain energy components, should be eliminated from the formulation for assuring convergence of results from a finite element model of the structural problems belonging to the class of constrained media elasticity.

Consistent mapping: Mapping of the strain/stress fields from $t$. . covariant natural system (where the element configuration is always undistorted) to the Cartesian system should not introduce any additional spurious constraints.

Edge-consistency: The tangential strain components which are continuous across the element boundary in the undistorted covariant natural system would remain continuous even after the necessary transformations; and the tangential strain components should be built from their corresponding tangential displacement components only.

Stress field-consistency: The terms in the strain and/or stress fields that do not participate in the strain energy computations (and hence in the displacement recovery) should be eliminated while recovering the corresponding strain and/or stresses in a displacement type formulation.

Variational correctness: The redistributed field should be orthogonal to the error introduced because of the field-redistribution with reference to the original field.

The ideal characteristics of a finite element formulation for general purpose applications and the paradigms/concepts required to achieve the same in a scientific manner are briefly shown in table 3.

Based on the consistency and correctness principles several linear and quadratic elements, free of locking and stress oscillation, are developed for 1-, 2- and 3dimensional applications.

\section{FEPACS - finite element package for analysis of composite structures}

FEPACS is a medium-sized general purpose package (i.e. about 20,000 lines of FORTRAN code) for the finite element analysis of isotropic, anisotropic and layered-composite structures. It has a family of simple, accurate and robust field-consistent elements. The package was initially built around the data and program organisation of SAP-IV, but recently, these have been modified and new solution capabilities are being introduced, to give FEPACS a character of its own.

The structural analysis program (SAP) is one of the earliest general purpose programs used for structural analysis through the finite element method. It was developed by Bathe et al (1974) under the sponsorship of many international organisations. The first version of SAP was released in September 1970 (Wilson 1970). The improved version, SAP-IV, which can be used for linear static and dynamic analysis of 3dimensional structures was released in 1974 (Bathe et al 1974). As it had been released with its source code in the public domain, it has served as the spring board for many other finite element packages which were improved versions of the original package. It is well known that SAP-IV has reasonably efficient solution capabilities and datahandling procedures that can solve large 3-dimensional systems. However, its main weakness is its very old, outdated element library based on mainly linear finite elements for isotropic structures. 
Table 3. Ideal characteristics of a finite element formulation.

\begin{tabular}{|c|c|}
\hline Characteristics & Paradigms/concepts/procedures \\
\hline $\begin{array}{l}\text { Discretisation errors should } \\
\text { vanish as the mesh is refined }\end{array}$ & Continuity and completeness \\
\hline $\begin{array}{l}\text { The element should be free of } \\
\text { locking and/or delayed convergence }\end{array}$ & $\begin{array}{l}\text { Field-consistency and } \\
\text { Variational correctness }\end{array}$ \\
\hline $\begin{array}{l}\text { Element should be able to give } \\
\text { variationally correct stress } \\
\text { distribution without any oscillations }\end{array}$ & $\begin{array}{l}\text { Field-consistency, } \\
\text { Stress field-consistency, } \\
\text { Variational correctness }\end{array}$ \\
\hline $\begin{array}{l}\text { Distorted geometry should not } \\
\text { disturb the element geometry }\end{array}$ & $\begin{array}{l}\text { Consistent mapping and } \\
\text { edge-consistency }\end{array}$ \\
\hline $\begin{array}{l}\text { The element should be free of } \\
\text { spurious zero energy mechanisms }\end{array}$ & $\begin{array}{l}\text { Consistency and correctness } \\
\text { Integration rules and Spectral } \\
\text { analysis }\end{array}$ \\
\hline $\begin{array}{l}\text { Element formulation should be scientific \& } \\
\text { should provide a priori methods of error } \\
\text { analysis (i.e. should not be based on } \\
\text { numerically adjusted factors, heuristic } \\
\text { arguments etc.) }\end{array}$ & $\begin{array}{l}\text { Consistency and correctness } \\
\text { paradigms supported by the } \\
\text { functional reconsistution } \\
\text { techniques }\end{array}$ \\
\hline $\begin{array}{l}\text { Element performance should be free } \\
\text { from its geometry and position in space }\end{array}$ & $\begin{array}{l}\text { Edge-consistency, appropriate } \\
\text { local coordinate system and } \\
\text { tensorial transformations }\end{array}$ \\
\hline
\end{tabular}

\subsection{Finite element library}

FEPACS has replaced this with the state-of-the-art field-consistent linear and quadratic 1-D, 2-D and 3-D element formulations - thus making the element library complete (Prathap et al 1989). The emphasis is on laminated anisotropic material structure (any other material constitution becomes a sub-set of this). A complete flow diagram representing the package is shown in figure 2 .

Each element in FEPACS utilises field- and edge-consistent stiffness matrices derived in a variationally correct fashion; consistent initial (thermal) strain representation; consistent stress resultant field representation; diagonal or consistent mass matrices; and geometric stiffness matrices which can be used for linear buckling analysis; material constitutive laws are derived assuming general orthotropic layers. The finite elements included in the library are given below.

(1) SPRING - 2-noded constant stiffness spring element,

(2) TRUSS - 2-noded linear truss element,

(3) BEAM2-T - 2-noded laminated linear Timoshenko beam element,

(4) BEAM2-E - 2-noded laminated linear classical beam element,

(5) BEAM3 - 3-noded laminated quadratic curved Timoshenko beam element (with taper and twist)

(6) PLAXTQ - family of plane strain, plane stress \& axisymmetric elements of triangular or quadrilater shapes (orthotropic),

(7) SHEL4 - 4-noded shear deformable laminated anisotropic Mindlin plate/ plane-shell element of quadrilateral shape with warping corrections, 


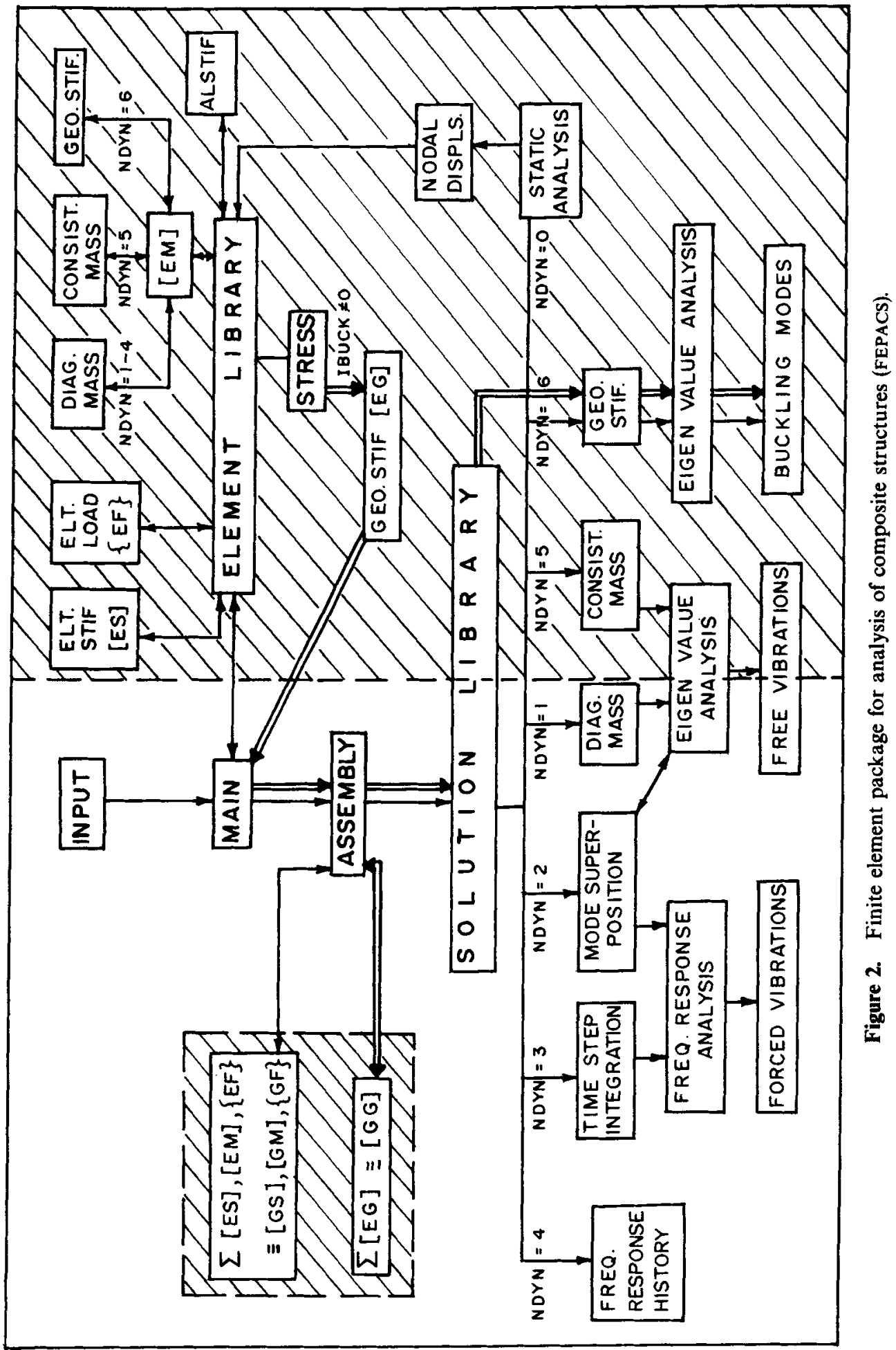


(8) SHEL8 - 8-noded quadrilateral laminated degenerated shell element,

(9) HEXA8 - 8-noded linear solid brick element (orthotropic),

(10) HEXA27 - 27-noded quadratic brick element (isotropic),

\subsection{Library of solution capabilities}

An eigenvalue solution routine based on the determinant search method has been added to FEPACS so that dynamic eigenvalue analysis using consistent mass matrices and buckling analysis of the linear structures can be carried out with the software. The solution routines can be efficiently used for both small-scale as well as large-scale problems using the respective in-core and out-of-core solution algorithms that are built in the program. The solution capabilities of FEPACS now include the following.

(1) Static analysis under thermomechanical loads-Gauss elimination solution routines based on banded assembled matrices are used;

(2) Natural frequency analysis - Both diagonal as well as consistent mass matrices can be used. Two eigenvalue solution routines, the determinant search method which is optimal for in-core solution and the subspace iteration method which is optimal for out-of-core solution, are available.

(3) Natural frequency analysis followed by response history analysis - Only diagonal mass matrices need to be used. Method of superposition of the natural modes and the forced modes is utilised to get the structural response to dynamic loads.

(4) Response history analysis using direct integration - Explicit time-step integration routines are used to get the structural response to dynamic loads without going through eigenvalue solution.

(5) Natural frequency analysis followed by response spectrum analysis.

(6) Buckling analysis - If the in-plane stresses are known a priori for each element, the analysis is done in a single run - construct the geometric stiffness matrices from the element in-plane stresses and solve for buckling loads and modes using the new eigenvalue solution routines. If they are not known (as in general structural analysis) the analysis is done in double runs - the in-plane stresses for each element are calculated from the first run of static analysis and the geometric stiffness matrices calculated from this static solution are then used for the second eigenvalue analysis.

\subsection{Other features}

Some of the other features specially incorporated in FEPACS to enhance its general purpose nature and its accessibility to users - for both regular analysis as well as for enhancement of the package with other capabilities include the following.

(a) Generalised data input and output structures so that integration of pre- and post-processors can be efficiently achieved.

(b) The program is organised in a highly modular fashion so that any new element or solution capability can be included with least difficulty.

(c) The whole program is modified to use only general FORTRAN-77 commands such that the package is highly portable except for the scratch file operations, while are kept in a separate module which is compiler-dependent. The package is currently available on a variety of platforms like PC 386/486, workstations, super-mini 
computers etc. with UNIX operating system, minimum 2 MB RAM and a FORTRAN compiler supporting scratch files of unlimited record length (only the scratch file allocations have to be modified). It can be loaded in any other operating system with similar support.

\section{Conclusions}

In this paper, many difficulties arising in displacement-type finite element formulation and the remedial measures offered for these have been reviewed. A general, complete and scientifically based procedure for formulating robust finite elements is provided. A general purpose finite element package (FEPACS) using a library of robust elements, developed along the lines presented here, is also reviewed. Though the emphasis is on the displacement-type finite formulations for structural analysis, the concepts discussed here are equally applicable to other types of finite element formulations applied to many other fields of engineering.

The authors are deeply indebted to Prof. R Narasimha, FRS, former Director of National Aerospace Laboratories, for his constant encouragement. They are also grateful to many colleagues - Dr H R Srinatha of Aeronautical Development Agency, Bangalore; Dr D H Bonde of Indian Space Research Organization, Bangalore and from the National Aerospace Laboratories, Drs G R Bhashyam, $S$ Viswanath, S Chandra, the late Mr C Ramesh Babu, M/s B Sudhakar, K Guruprasad, S Nagaraj, V Baskar, Shaik Cheman, J Durga Prasad and Ms B R Shashirekha who have supported or worked on the development of FEPACS at various times.

\section{References}

Babu C R 1985 Field-consistency in the finite element formulation of multi-strain-field problems in structural mechanics. Ph D Thesis, IIT Madras

Bathe K J, Dvorkin E L 1985 A four-node plate bending element based on Mindlin/Reissner plate theory and a mixed interpolation. Int. J. Numer. Methods Eng. 21: 367-383

Bathe K J, Wilson E L, Peterson F E 1974 SAP IV - A structural analysis program for static and dynamic response of linear systems. College of Engineering, University of California, Berkeley

Belytschko T, Liu W K, Ong J S J 1984a Nine node Lagrange shell elements with spurious mode control. Proc. AIAA/ASME. Struct. Dyn. Conf., Palm Springs

Belytschko T, Liu W K, Ong J S J, Lamb D 1985 Implementation and application of a nine-noded Lagrangian shell element with spurious mode control. Comput. Struct. 20: $121-128$

Belytschko T, Ong J S J, Liu W K 1984b A consistent control of spurious singular modes in the 9-node Lagrangian element for the Laplace and Mindlin plate equations. Comput. Methods Appl. Mech. Eng. 44: 269-295

Bose C J, Kirkhop J 1984 Least squares strain smoothing for the eight-node serendipity plane stress element. Int. J. Numer. Methods Eng. 20: 1164-1166

Chandra S, Prathap G 1989 A field-consistent formulation for the 8-noded solid finite element. Comput. Struct. 33: 345-355

Cook R D 1975 Avoidance of parasitic shear in plane element. J. Struct. Div. ASCE. 93: 43-66 
Cook R D, Malkus D S, Plesha M E 1981 Concepts and applications of finite element analysis 3rd edn (New York: John Wiley \& Sons)

Crisfield M A 1984 A quadratic Mindlin element using shear constraints. Comput. Struct. 18: 833-852

Doherty W P, Wilson E L, Taylor R L 1969 Stress analysis of axisymmetric solids using higher order quadrilateral elements, SESM Report 69-3, Dept. of Civil Eng., University of California, Berkeley

Donea J, Lamain L G 1987 A modified representation of transverse shear $C^{0}$ quadrilateral plate elements. Comput. Methods Appl. Mech. Eng. 63: 183-207

Fried I 1974 Finite element analysis of incompressible material by residual energy balancing. Int. J. Solids Struct. 10: 993-1002

Fried I 1975 Finite element analysis of thin elastic shells with residual energy balancing and the role of the rigid body modes J. Appl. Mech. 42: 99-104

Gilewski W, Radwanska M 1991 A survey of finite element models for the analysis of moderately thick shells. Finite Element Anal. Design 9: 1-21

Heppler G R, Hansen J S 1987 Timoshenko beam finite elements using trignometric basis functions. $A I A A$ J. 26: 1378-1386

Hinton E, Huang H C 1986 A family of quadrilateral Mindlin plate elements with substitute shear strain fields. Comput. Struct. 23: 409-431

Hoppe V 1984 Errors in the finite element solutions introduced by mapping in isoparametric elements. Proc. of the 4th world congress and exhibition on finite element methods (Wimborne, Dorset: Robinson and Associates)

Hoppe V 1985 Faith and fact in finite elements. Finite Element News 2: 12-15

Huang H C, Hinton E 1984 A nine-code Lagrangian Mindlin plate element with enhanced shear interpolation. Eng. Comput. 1: 369-379

Hughes T J R 1987 The finite element method: Linear static and dynamic finite element analysis (Englewood Cliffs, NJ: Prentice Hall)

Hughes T J R, Cohen M, Haroun M 1978 Reduced and selective integration techniques in the finite element analysis. Nucl. Eng. Design 46: 206-222

Hughes T J R, Taylor R L, Kanoknukulchal W 1977 A simple and efficient finite element for plate bending. Int. J. Numer. Methods Eng. 11: 1529-1543

Jang J, Pinsky P M 1988 Convergence of curved shell elements based on assumed covariant strain interpolations. Int. J. Numer Methods Eng. 26: 329-347

MacNeal R H 1982 Derivation of element stiffness matrices by assumed strain distributions. Nucl. Eng. Design 70: 3-12

Malkus D S, Hughes T J R 1978 Mixed finite element method - reduced and selective integration techniques: unification of concepts. Comput Methods Appl. Mech. Eng. 15: 68-81

Mohr G A 1982 Application of penalty functions to a curved isoparametric axisymmetric thick shell element. Comput. Struct. 15: 685-690

Naganarayana B P 1991 Consistency and correctness principles in quadratic displacement type finite element. $\mathrm{Ph} \mathrm{D}$ thesis, Indian Institute of Science, Bangalore

Naganarayana B P, Prathap G 1989a Displacement and stress predictions from field- and line- consistent versions of the 8-node Mindlin plate element. Comput. Struct. 33: 1095-1106

Naganarayana B P, Prathap G 1989b Force and moment corrections for the warped four-node quadrilateral plane shell element. Comput. Struct. 33: 1107-1115

Naganarayana B P, Prathap G 1991 Field consistency analysis of 27-noded hexahedral elements for constrained media elasticity. Finite Elements Anal. Design 9: 149-168

Naganarayana B P, Prathap G, Dattaguru B, Ramamurthy T S 1992 A field-consistent and variationally correct representation of transverse shear strains in the nine-noded plate element. Comput. Methods Appl. Mech. Eng. 97: 355-374

Noor A K, Anderson C M 1982 Mixed models and reduced-selective integration displacement models for nonlinear shell analysis. Int. J. Numer. Methods Eng. 18: 1429-1454

Noor A K, Hartley S J 1977 Nonlinear shell analysis via mixed isoparametric elements. Comput. Struct. 7: 615-626

Noor A K, Peters J M 1981 Mixed models and reduced-selective integration displacement models for nonlinear analysis of curved beams. Int. J. Numer. Methods Eng. 17: 615-631

Ojalvo I U 1974 Improved thermal stress by finite element methods. AIAA J. 12:1131-1132 
Oleson J F 1983 Field-redistribution in finite elements - a mathematical alternative to reduced integration. Comput. Struct. 17: 157-159

Park K C 1984 Symbolic Fourier analysis procedures for $\mathrm{C}-\mathrm{O}$ finite elements. Innovative methods for nonlinear analysis, pp. 269-293

Pawsey S E, Clough R W 1971 Improved numerical integration of thick shell finite elements. Int. J. Numer. Methods Eng. 3: 545-586

Pittr J, Hartl H 1980 Improved stress evaluation under thermal load for simple finite elements. Int. J. Numer Methods Eng. 15: 1507-1515

Prathap G 1993 Finite element method in structural mechanics (Dordrecht: Kluwer)

Prathap G 1985a A $C^{0}$ continuous four-noded cylindrical shell element. Comput. Struct. 21: 995-999

Prathap G 1985b The curved beam/deep arch/finite ring element re-visited. Int. J. Numer. Methods Eng. 21: 389-407

Prathap G 1985c The poor bending response of the four-noded plane stress quadrilateral element. Int. J. Numer. Methods Eng. 21: 825-835

Prathap G 1986 Field-consistency - Toward a science of constrained multi-strain-field finite element formulations. Sadhana 9: 345-353

Prathap G 1988 The variational basis for least squares field-redistribution of strain functions in the finite element formulations of constrained media elasticity. TM ST 8801, National Aeronautical Laboratory, Bangalore

Prathap G G 1994 Locking, rank and non-singularity of penalty linked stiffness matrix and consistency of strain field Comput. Struct. (to be published)

Prathap G, Babu C R 1986a An isoparametric quadratic thick curved beam element. Int. J. Numer. Methods Eng. 23: 1583-1600

Prathap G, Babu C R 1986b Field-consistent strain interpolations for the quadratic shear flexible beam element. Int. J. Numer. Methods Eng. 23: 1973-1984

Prathap G, Babu C R 1987 Field-consistency and violent stress oscillations in the finite element method. Int. J. Numer. Methods Eng. 24: 2017-2033

Prathap G, Bhashyam G R 1982 Reduced integration and the shear-flexible beam element. Int. J. Numer. Methods Eng. 18: 195-210

Prathap G, Dattaguru B, Naganarayana B P 1989 Development of general purpose finite element package for structural analysis of isotropic, anisotropic and layered-composite structures: Project Proposal to AR \& DB. PP ST 8907, National Aeronautical Laboratory, Bangalore

Prathap G, Naganarayana B P 1988 Consistency and orthogonality requirements and accurate stress recovery for the assumed strain eight-noded quadrilateral plate bending elements. TM ST 8805, National Aeronautical Laboratory, Bangalore

Prathap G, Naganarayana B P 1990a Consistent force resultant distributions in displacement elements with varying sectional properties. Int. J. Numer. Methods Eng. 29: 775-783

Prathap G, Naganarayana B P 1992 Field-consistency rules for a 3-noded shear-flexible beam elements under non-uniform isoparametric mapping. Int. J. Numer. Methods Eng. 33: 649-664

Prathap G, Naganarayana B P 1994 Consistent thermal stress evaluation in finite elements (to be published)

Prathap G, Naganarayana B P, Somashekar B R 1988 Field-consistency analysis of the isoparametric eight-noded plate bending element. Comput. Struct. 29: 857-873

Prathap G, Nirmala K 1990 Finite element formulations of constrained media elasticity A bibliography. Finite Elements Anal. Design 7: 253-270

Prathap G, Somashekar B R 1988 Field- and Edge- consistency synthesis of a four-noded quadrilateral plate bending element. Int. J. Numer. Methods Eng. 26: 1693-1708

Prathap G, Subramanian G, Babu C R 1986 Stress oscillations in plane stress modelling of flexure - a field-consistency approach. Int. J. Numer. Methods Eng. 24: 711-724

Prathap G, Viswanath S 1983 An optimally integrated four-noded quadrilateral plate bending element. Int. J. Numer. Methods Eng. 19: 831-840

Simo J C, Hughes T J R 1986 On the variational foundations of assumed strain methods. $J$. Appl. Mech. 53: 51-54

Stolarski H, Belytschko T 1981 Membrane locking and reduced integration for curved elements. J. Appl. Mech. 49: 172-178 
Stolarski H, Carpenter N, Belytschko T 1985 A Kirchoff-mode method for C-O bilinear and serendipity plate elements. Comput. Methods Appl. Mech. Eng. 50: 121-145

Tang L, Chen W, Liu Y 1984 Formulation of quasi-conforming element and Hu-Washizu principle. Comput. Struct. 19(1-2): 247-250

Tessler A, Dong S B 1981 On a hierarchy of conforming Timoshenko beam elements. Comput. Struct. 14: 335-344

Walz J E, Fulton R E, Cyrus N J, Eppink R T 1970 Accuracy of finite element approximations to structural problems. NASA TN-D 5728

Wilson E L 1970 SAP - A general structural analysis program. SESM Report 70-20, Dept. of Civil Engineering, University of California, Berkeley

Wilson E L, Taylor R L, Doherty W P, Ghabussi T 1973 Incompatible displacement models. Numer. Comput. Methods in Struct. Mech. (ed. S T Ferves et al), Academic Press, pp. 43-57

Zienkiewicz O C 1977 The finite element method 3rd edn (London: McGraw Hill)

Zienkiewicz O C, Hinton E 1976 Reduced integration, function smoothing and non-conformity in finite element analysis. J. Franklin Inst. 302(56): 443-461

Zienkiewicz O C, Taylor R L, Too J M 1971 Reduced integration techniques in general analysis of plates and shells. Int. J. Numer. Methods Eng. 3: 275-290 